\title{
Scoliosis: current overview and treatment considerations
}

\author{
Elton Gomes da Silva', Hamilton D. Kojo², \\ Andrei Fernandes Joaquim ${ }^{3}$, Christopher I. Shaffrey ${ }^{4}$ \\ Department of Neurology, State University of Campinas (Unicamp), Campinas, SP, Brazil. \\ Department of Neurological Surgery, University of Virginia (UVA), Charlottesville, VA, USA.
}

\begin{abstract}
Scoliosis presents with lateral curvature of the spine greater than 10 degrees in $x$-ray and it can be associated with others deformities of the curvature of the spine. The management of this deformity is dependent of the classification and clinical presentation of the patient. This paper purposes to discuss to the initial evaluation of patients with scoliosis, distinguishing the different types of scoliosis and the management of patients with this diagnosis.

\section{KEYWORDS}

Scoliosis, classification, treatment.

\section{RESUMO}

Escoliose: conceitos atuais e considerações terapêuticas

A escoliose apresenta-se como uma curvatura lateral da coluna maior que 10 graus na radiografia e pode ser associada a outras deformidades da curvatura da coluna. O manejo terapêutico dessa deformidade é dependente da classificação e apresentação clínica do paciente. Este artigo se propõe a discutir a evolução inicial de pacientes com escoliose, distinguindo os diferentes tipos dessa anormalidade e o manejo dos pacientes.
\end{abstract}

\section{PALAVRAS-CHAVE}

Escoliose, classificação, tratamento.

\section{Introduction}

The spinal deformity with lateral curvature greater than 10 degrees is named scoliosis. A new challenge for neurosurgeons who deal with spine surgery is to treat patients with this abnormality. In the past, it was an exclusive orthopedic field in medicine; however, more and more neurosurgeons that perform spine surgeries are responsible for the management of these patients. In this paper, we review the initial evaluation of patients with scoliosis, discussing the classification and surgical approaches.

The normal anatomy of the spine is represented by a characteristic cervical and lumbar lordotic curvature and thoracic and sacral kyphosis observed in the sagittal plane. A curvature in the coronal plane causing a lateral shift in vertebral body alignment is abnormal. This spinal deformity in the coronal plane is named scoliosis, and any deformity in the sagittal plane can result in unusual kyphosis, hyperlordosis or a combination kyphoscoliosis with a rotational component.

The Scoliosis Research Society has defined scoliosis as a spinal deformity with lateral curvature greater than 10 degrees in magnitude as measured with standing $\mathrm{x}$-rays with the Cobb technique. ${ }^{19}$ The curvature of the spine is a complex three-dimensional rotational deformity that affects the spine in the coronal, sagittal and axial (vertebral rotation) planes and, therefore, its treatment should be directed to the three features to improve patient's function. ${ }^{13,14}$

1 Resident of Neurosurgery - Department of Neurology, State University of Campinas (Unicamp), Campinas, SP, Brazil.

2 Department of Neurological Surgery, University of Virginia (UVA), Charlottesville, VA, USA. Complex Spine Fellow, UVA.

3 Neurosurgeon - Campinas, SP; PhD student, Department of Neurology, Unicamp.

4 Department of Neurological Surgery, UVA. Professor of Neurological Surgery, UVA. 


\section{General considerations}

Generally, the scoliosis can occur as a result of a congenital spinal malformation, from spinal cord intrinsic anomalies (tumor or syrinx), a neuromuscular disorder, facet or disc degeneration, neurofibromatosis, connective tissue disease, skeletal dysplasia, iatrogenic, or it presents as a unknown cause. , $^{4,12,20}$

The clinical presentation of this deformity is different in adults than in children, as in the prevalence, clinical evaluation, radiographic features, therapeutic process, and prognosis, therefore it is necessary to have a distinct classification for both groups. In adults, the rates of scoliosis related range from $2 \%$ to $32 \%$, increasing proportionally in relation to age. In adolescents, the prevalence reaches $2 \%-3 \%$ of this population, and it is less common in younger children. ${ }^{10,40}$ The clinical presentation in adults commonly begin with back and leg pain, and neurological symptoms that causes disability; these symptoms occur in conjunction with muscular fatigue and spasm, trunk imbalance, facet arthropathy, disc degenerative disease, stenosis of the spinal and/ or foraminal canal and, as the curve progress slowly, cosmetic aspects are less common complaints. $7,32,34,37$ In childhood, progression of the curve is significant and alteration of cosmetic and mental image are more important than symptoms of pain. ${ }^{30,37}$

The initial evaluation of the patient with any suspect spinal deformity begins with posteroanterior (PA) and lateral 36-inch $\mathrm{x}$-rays that shows occiput proximally and the femoral heads distally to evaluate the regional and global alignment of the spine and to define the Cobb angles, the central sacral vertical line, the neutrally rotated vertebrae, the stable vertebrae, the apical deviation, kyphosis and lordosis, sagittal balance, with evaluation for mixed deformities which are required in the moment of the treatment, and it is the first choice for diagnosis and follow-up of patients. ${ }^{3,36}$ PA projections with side-bending can be done for assessment of the flexibility and dynamic spinal instability that can influence in the decision about treatment, because larger curves have less flexibility than smaller curves, and the flexibility decreases by $10 \%$ with every 10 degree increase in coronal deformity beyond 40 degrees and also flexibility decreases by $5 \%$ to $10 \%$ with each decade of life. ${ }^{15}$

Computed tomographic imaging (CT) can help to define bone anatomy and dimensions and underlying anomalies unidentified in the x-ray. ${ }^{9}$ Magnetic resonance imaging (MRI) is indicated in neurological abnormalities, severe pain, early-onset scoliosis with curves greater than 20 degrees (studies show around $20 \%$ of abnormalities of the neural axis in this population), atypical curve patterns (e.g., left thoracic curves, short angular curves, congenital deformities, or curves greater than 70 degrees), curve with rapid progression (more than one degree/month), evaluating for neoplastic process in neurofibromatosis patients, deformity associated with myelomeningocele and absence of apical lordosis in idiopathic scoliosis. ${ }^{3,7,16,32}$ If there is a contraindication to undergoing a MRI, a myelogram with a post-myelogram CT scan is useful. ${ }^{17}$

Successful management of these deformities requires one to define, to anticipate and potentially control the growth of the vertebral elements, with operative or nonoperative treatment. If surgical treatment is necessary, attention must be paid to documenting multiple discussions with patients and careful assessment of expectations as morbidity and disability risk related to surgery may be more than what is experienced preoperatively from spinal deformity. ${ }^{29}$

\section{General classification}

Primarily, scoliosis can be grouped in two distinct classes: 1) non-structural; and 2) structural.

1) Non structural: it refers to whose measured curve in the coronal plane in which the Cobb measurement corrects past zero on supine lateral side bending x-ray. It can have many etiologies, like postural, hysteric, inflammatory and compensatory.

2) Structural: it designates those curves the coronal plane in which the Cobb measurement fails to correct past zero on supine maximal voluntary lateral side bending x-ray. It means that the curve is nonflexible. For instance, in adolescent idiopathic scoliosis, specific criteria of structural and non structural can be applied according to treatment plan. ${ }^{23}$ Etiology of the structural's curve can include: neuromuscular diseases, congenital, idiopathic (infantile, juvenile, adolescent and adult) and secondary degenerative disease.

In this part, we briefly describe the main characteristic of each etiological group of structural scoliosis.

\section{Neuromuscular scoliosis}

Defined as scoliosis in a child with any known nerve or muscle disease, such as cerebral palsy, muscular dystrophy or spinal cord injury leading to the scoliosis with curves generally rigid and including the pelvis. The Scoliosis Research Society classifies these neuromuscular disorders in neuropathic and myopathic types. The neuropathic group has been subclassified further into those with upper (cerebral palsy, syringomyelia, 
spinal cord trauma) and lower (poliomyelitis and spinal muscular atrophy) motor neuron lesions. The myopathic group includes conditions such as arthrogryposis, muscular dystrophy, and other forms of myopathy. 1,20,32 Progression occurs much more frequently than in idiopathic scoliosis, most likely as a result of asymmetric muscle tone and strength. However the pathophysiology of neuromuscular scoliosis is not well understood, because besides muscle weakness, some cases may be accompanied by spasticity and others by flaccidity. ${ }^{1}$

The goals of deformity correction are a normal sagittal and coronal alignment, shoulders level and head centered over pelvis, preservation of as many motion segments as possible, and fusion and arrest of progression. Generally, construct for correction of neuromuscular scoliosis includes many vertebrae to provide stability to overcome the deforming forces and allow early mobilization without a bracing. ${ }^{30}$

\section{Congenital scoliosis}

Congenital abnormalities of the spine resulting from the abnormal vertebral development occurring during the fourth to sixth weeks of gestation, with abnormal lateral curvature present at birth. It may be associated with other neural anomalies in $10 \%$ to $20 \%$ of patients, and other developmental abnormalities such as cardiac and renal abnormalities may be present as well. ${ }^{1,28}$

Congenital scoliosis is rare (overall incidence is unknown, but it has been estimated at 0.5 per 1000 patients in the thoracic spine), and cannot present a significant abnormality until the patient reaches adolescence. ${ }^{28}$ Generally, it can be classified as failure of formation (like wedge vertebra or hemivertebra), failure of segmentation (unilateral unsegmented bar or block vertebra) or mixed. ${ }^{1,20}$ The most severe form of scoliosis is associated with the presence of hemivertebra, with rapidly progressive curvature. Nevertheless, it is difficult to determine which curves will progress rapidly. In general, $25 \%$ progress rapidly, $25 \%$ do not progress and half display slow progression. The cervicothoracic and lumbosacral junctional areas produce larger deformities than other levels of the spine. ${ }^{25}$

Children can be followed initially every three months to evaluate for curve progression. If this remains stable, the follow-up imaging can be done every six months. When they reach adolescence, however, it is recommended a follow-up more often, approximately every three to four months. ${ }^{1}$

Conservative treatment consists in the use of bracing, which is very limited, and rarely used, because many these deformities are rigid and progressive and thus require surgery. Bracing would be most helpful in flexible curves, such as those accompanying scattered anomalies separated by mobile segments or curves in which the anomalies are at the upper end of the curve and the lower portion contains mobile segments, and a compensatory curve composed of normal vertebrae and discs, if progressive and flexible., ${ }^{1,32}$

Surgery is indicated when the curve progression occurs regardless of bracing or that curve likely will continue to progress. Operative management may include in situ surgical fusion, anterior/posterior hemiepiphysiodesis, hemivertebra excision, in addition to fusion techniques, which can be used in children at a young age with the use of growing rods that are lengthened by distraction until the definitive final arthrodesis with instrumentation can be performed..$^{21,38,42}$

\section{Idiopathic scoliosis}

In this group, there is a structural curve for which the cause has not been established. No evidence of underlying physical or radiographic pathology is present (Scoliosis Society).

It is the most common type of scoliosis in skeletally immature population, comprising approximately $0.5 \%$ of all patients with scoliosis; it has had a higher incidence in adolescence, but it may begin at a point prior to skeletal maturity and may be found incidentally or present with symptoms in adult patients. Although it does not present a definite cause, often it may be associated with dysplasia of pelvis, plagiocephaly and congenital heart disease, in addition to genetic factors appear to be involved, but its inheritance pattern is still not resolved. ${ }^{20,22,24,40}$

The most classical classification, defined by the Scoliosis Research Society, has subdivided idiopathic scoliosis (IS) into four subgroups based on patient's age:

- Infantile IS - children from birth to 2 years 11 months of age with IS are classified as having infantile IS;

- Juvenile IS - from 3 to 9 years 11 months;

- Adolescent IS - children between 10 years and 17 years 11 months;

- Adult IS - is deformity presenting after 18 years of age.

But it is important to remember that age is an arbitrary number, since it is not possible to specify how long a patient may have had a particular curve before it was diagnosed, then the recent tendency is to classify patients based simply on early onset $(<10$ year-old $)$ versus late-onset (adolescent) scoliosis, since works show the growth velocity is relatively constant between five and 10 years of age which is proven by the relative rarity of this disturbance in juvenile form. ${ }^{32,37,40}$ Before 10 years of age, the rate of curve progression is of 1-3 degrees per year, whereas it is 4.5-11 degrees per year 
after the age of 10 years. Progression is defined as a curve increasing greater than five degrees on consecutive follow-up imaging. ${ }^{20}$

\section{Childhood idiopathic scoliosis (infantile, juvenile and adolescent)}

Natural history studies on infantile scoliosis have shown that many cases resolve spontaneously, particularly those younger than one year of age, and in some studies approximately $90 \%$ of cases resolved at skeletal maturity without surgical intervention. However, the curves which progress can be very difficult to treat because of the significant remaining growth and the negative effect of the curve progression on cardiopulmonary and gastrointestinal tract development, so careful and frequent monitoring of these patients is necessary. ${ }^{11,22,40}$ Potential indicators of progressive deformity include a rib-vertebra angle difference greater than 20 degrees, double curve pattern and overlapping of the apical vertebra by the apical rib, very skeletally immature patients, and curves greater than 25 degrees.,20

In general, the treatment is done on the basis of consensus in relation at the progression before overall skeletal maturity. Curves less than 20 to 25 degrees are observed by clinical and radiographic exams regularly at past their peak height velocity. ${ }^{3}$

The non-operative treatment is performed with the use of bracing or cast. Its use is based on uncontrolled observational studies with variable and not standardized results, so that its use remains controversial. The goal of bracing therapy, generally, is allow continued trunk growth in a child with scoliosis who is likely to need surgery but still has significant growth potential remaining. ${ }^{2,20,32}$ Commonly, bracing is used for patients with early onset IS with a curve magnitude of 25 to 45 degrees and may be used on curves less than 25 degrees if the patient has a high probability of curve progression. In late-onset scoliosis, bracing can be used in curves greater than 20 degrees, those that progress more than five degrees or curves between 25 and 40 degrees with significant growth remaining, regardless of documented progression, however its use should be discontinued if the curve reaches a surgical dimensions (45-50 degrees) or as the skeletal maturity is achieved. ${ }^{20,32}$ However, it is important to remember the bracing does not correct the scoliosis, only prevents the curve progression, permitting stability to the potential magnitude of the deformity. The control of scoliotic curves when the apex is at or below T7 is most commonly done using a thoracolumbosacral orthosis (e.g., Charleston, Wilmington and Boston braces) and a cervicothoracolumbosacral orthosis (e.g., Milwaukee brace) is used when the scoliotic curve apex is above $\mathrm{T} 7$. The time recommended for daily application spine bracing ranges from eight to 23 hours, and patients would be followed every four to six months with radiographic image to assess the progression. If the curves are solved, the range can be extended for one year, but the child should be followed until skeletal maturity. If complete stabilization is attained before of prepubertal growth spurt, relapse during adolescence is very unlikely. When there is no evidence of curve progression, bracing can be discontinued. ${ }^{32}$ Treatment of infants (until 18 months of age) is primarily by serial molded body casts followed by bracing. The casts are applied and removed under general anesthesia at six to 12 weeks intervals. The maximum correction possible is reached by 18 months of age, and at this point is placed a brace., ${ }^{2,32}$

When these methods fail or are contraindicated, surgical intervention should be considered. The goal is to improve the sagittal and coronal balance, to prevent the curve progression, to avoid functional limitations, and to decrease the number of fused segments. ${ }^{20}$

Once the curve reaches 45 degrees in early-onset scoliosis, surgery would be considered, taking into account that the growth spurt has not occurred, and a solid fusion would prevent additional longitudinal growth and potential adult height scope; in addition it can result in crankshaft phenomenon. Strategies for operative treatment in these patients include techniques for nonfusion instrumentation, single and dual growing rods with claw foundations proximally and distally, vertical expandable prosthetic titanium rib (VEPTR), clipping of the vertebral body and use of multiple molds brokers, and no technique is quite effective, however allow a periodic growth until the child reaches an appropriate size and maturity for definitive arthodesis. 2,18,32,39 However, its use is controversial because of high rates of complications and reports questioning whether the amount of growth facilitated by its use justifies the surgical procedures for periodic lengthening required.

Surgery for late-onset scoliosis is recommended for curves greater than 45 degrees if the child has not reached skeletal maturity, and above 50 degrees mature young, because, according to studies, progression of curves of this magnitude continue to occur in adulthood in $68 \%$ of cases. ${ }^{40}$ There is no consensus on the best method of fusion with instrumentation, however studies show that in the posterior fusion techniques, greater correction of Cobb angles occurred with the use of constructs with pedicle screw. ${ }^{32,40}$

\section{Adult idiopathic scoliosis}

Approximately $2 \%$ to $4 \%$ of adults under 45 years of age have scoliosis, most of which have idiopathic scoliosis. ${ }^{27}$ Usually asymptomatic deformities are not treated in adults, unless a substantial magnitude curve 
with a virtual certainty of progression is identified in a relatively young adult. They should be educated about the potential signs and symptoms of progression with observation at regular intervals of time (six to 12 months initially and if the progression is minimal or nonexistent, it can be extended). ${ }^{2,35}$

Conservative treatment is essential before surgery is considered, including encouragement of overweight patients to lose weight with the help of a structured weight loss program, physical therapy, mainly focused on extension-based exercises. However these techniques are used to minimize the symptoms and not a mean of correcting the curves, and the choice between operative and nonoperative treatment is complex and multifaceted. ${ }^{17,33}$ Bracing therapy for a long time can lead to unpleasant effects such as muscle atrophy and early saturation and weight loss, and reserved only for the management of scoliosis in patients who are not candidates for surgery or for short periods (six to twelve weeks) of treatment in symptomatic cases.

The indications for surgery are intractable pain (axial, radicular, or both, and leg pain) related to spinal curvature, documented curve progression, balance decompensation, presence of cosmetically unacceptable deformity to the patient, but only severe radiculopathy, radicular weakness and more sagittal imbalance proved to be related to a better indication for surgery. $731,33,35$ With stiffer curves in adults than in adolescents, surgical correction is more difficult and associated with a higher rate of complications. So well-established goals should be addressed to prevent the curve progression and/or correction of the curve, improvement in pain and improved function and achieving a appropriate sagittal and coronal balance, with the principle of obtaining a solid fusion with the spine in a three-dimensional balanced state to prevent further deformity. ${ }^{17,20}$ Wires, hooks and pedicle screws have been used in surgical correction of this deformity, and the pedicle screws have the advantage of the ability to achieve stronger fixation than that with hooks and to improve the ability to enhance or maintain lumbar lordosis. Besides the instrumentation by the posterior approach, it can be associated with anterior surgery and/or osteotomies to achieve greater degrees of segmental correction beyond that allowed by the curve`s inherent flexibility., ${ }^{2,17}$

\section{Adult degenerative scoliosis}

Degenerative lumbar scoliosis is a rotational disorder that creates hypolordosis and it is associated at a compensatory thoracic relatively flexible curve typically less than 30 degrees. Common radiographic findings include degenerative disc disease, most com- monly at L3-L4 and L4-L5 obliquity, in addition at rotational subluxation or lateral translation at L3-L4 and L4-L5 obliquity. ${ }^{8}$ Usually, patients present with symptoms of chronic low back pain ( $90 \%$ of patients report pain as a primary complaint), in addition to neurogenic claudication and severe axial pain, and that is caused by spinal or foraminal canal stenosis associated with degenerative structural deformity. 12,43

Initial treatment for patients with pain and even for those patients with axial or mechanical low back pain, is with nonsteroidal anti-inflammatory drugs (NSAIDs), muscle relaxants and analgesics. They can be augmented with epidural steroid injections, nerve roots blocks, and facet injections when symptoms persist. ${ }^{5,34}$ Adjuvant therapy used also includes physical therapy and/or aquatic therapy, and they may help with reconditioning and to maintain muscle tone. Bracing does not alter the curve progression, but its stabilizing effect may help relieve pain, however, it should not be used worn for extended periods of time to prevent muscle atrophy and deconditioning. ${ }^{5}$

Surgery should be offered to patients with deformity related pain who have failed with conservative treatment, particularly those with a tendency to curve progression (e.g., thoracic curves $>50-60$ degrees and lumbar curves $>40$ degrees), besides the associated kyphosis $>60$ degrees in the thoracic spine and five degrees in the lumbar spine, or persistent neurological deficit (e.g., radiculopathy or neurogenic claudication). The latter is becoming more prevalent, likely due at an aging population, it should be prioritized to the decompression of neural elements in these patients. Furthermore, associated with correction of deformity, it should be given attention to the adequacy of sagittal balance, since loss of lumbar lordosis is associated with poor evolution. ${ }^{5}$

\section{Operative considerations}

When operative management is decided, preoperative clearance with thorough medical and anesthetic considerations must be performed due to the significant risk of morbidity associated with curve correction for optimal result. An important feature in this view is the need for consideration of lung volume in the perioperative management, and it is essential to do formal pulmonary function tests to assess lung volumes and compliance prior to surgery in patients with relative hypokyphosis of the thoracic spine or a scoliosis curve greater than 80 degrees. ${ }^{20}$ The blood loss in surgery for scoliosis correction may exceed $50 \%$ of the blood volume of the patient, depending on approach, surgical 
time, use of bone graft taken from the hip, increased intra-abdominal pressure. Modifications in surgical technique to reduce blood loss include, decreasing surgical time, staging correction procedure (e.g., performing anterior lengthening posterior followed by pedicle screw fixation after a few days), positioning patients to prevent venous congestion, neuromuscular blockers to relax the abdominal muscles, preoperative autologous blood donation, acute infusion of shed blood through the use of a cell saver. ${ }^{4}$

Although rare, neurological damage is the most severe injury that can occur as a complication of surgery to correct scoliosis, so it is recommended that intraoperative monitoring of somatosensory and motor evoked potentials to minimize the risk of neurological complications. ${ }^{4,26}$

Complications of surgery include postoperative neurological deterioration, postoperative wound infection, persistent cerebrospinal fluid leak, thromboembolic events, implant failure, adjacent level kyphosis post instrumentation, adjacent segment degeneration, crankshaft phenomenon and pseudoarthrosis. The latter is responsible for up tol one quarter of non-union in operated patients. ${ }^{4-7,17,26,30}$

\section{Conclusion}

Scoliosis is a deformity in the coronal plane of the spine, associated with alterations of the axial and sagittal plane, therefore it must be viewed as a complex three-dimensional rotational deformity. Several factors are involved in the decision making for patients. Patient care is best directed by well trained, specialized spine surgeons who through multiple visits and years of positive relationship with the patient eventually reach a mutual satisfaction and expectations from surgical or directed conservative treatment.

\section{References}

1. Aliabadi H, Grant G. Congenital thoracolumbar spine deformities. Neurosurgery. 2008;63:78-85.

2. Angevine PD, Deutsch H. Idiopathic scoliosis. Neurosurgery. 2008;63:86-93.

3. Angevine PD, Kaiser MG. Radiographic measurement techniques. Neurosurgery. 2008;63:40-5.

4. Bilsky MH, Boachie-Adjei, Shields CB, Baldwin N. Thoracic and lumbar deformities. In: Benzel EC, editor. Spine surgery: techniques, complication avoidance, and management. Philadelphia: Elsevier Churchill Livingstone; 2005, p. $796-823$.
5. Birknes JK, White AP, Albert TJ, Shaffrey Cl, Harrop JS. Adult degenerative scoliosis: a review. Neurosurgery. 2008;63:94-103.

6. Bradford DS, Lonstein JE, Moe JH, Ogilvie JW, Winter RB Moe's textbook of scoliosis and other spinal deformities. Philadelphia: W.B. Saunders; 1989.

7. Bradford DS, Tay BK, Hu SS. Adult scoliosis: surgical indications, operative management, complications, and outcomes. Spine. 1999;24:2617-29.

8. Bridwell $\mathrm{KH}$. Selection of instrumentation and fusion levels for scoliosis: where to start and where to stop. J Neurosurg Spine. 2004;1:1-8.

9. Bush $\mathrm{CH}, \mathrm{Kalen} \mathrm{V}$. Three-dimensional computed tomography in the assessment of congenital scoliosis. Skeletal Radiol. 1999;28:632-7.

10. Carter OD, Haynes SG. Prevalence rates for scoliosis in US adults: results from the first National Health and Nutrition Examination Survey. Int J Epidemiol. 1987;16:537-44.

11. Ceballos T, Ferrer-Torrelles M, Castillo F, FernandezParedes E. Prognosis in infantile idiopathic scoliosis. J Bone Joint Surg (Am). 1980;62:863-75.

12. Davies A, Saifuddin A. Imaging of painful scoliosis. Skeletal Radiol. 2009;38:207-23.

13. Deacon P, Archer IA, Dickson RA. The anatomy of spinal deformity: a biomechanical analysis. Orthopedics. 1987;10:897-903.

14. Deacon P, Dickson RA. Vertebral shape in the median sagittal plane in idiopathic thoracic scoliosis: a study of true lateral radiographs in 150 patients. Orthopedics. 1987;10:893-5.

15. Deviren V, Berven S, Kleinstueck F, Antinnes J, Smith JA, Hu SS. Predictors of flexibility and pain patterns in thoracolumbar and lumbar idiopathic scoliosis. Spine. 2002;27:2346-9.

16. Dobbs MB, Lenke LG, Szymanski DA, Morcuende JA, Weinstein SL, Bridwell KH, et al. Prevalence of neural axis abnormalities in patients with infantile idiopathic scoliosis. J Bone Joint Surg (Am). 2002;84:2230-4.

17. Heary RF, Kumar S, Bono C. Decision making in adult deformity. Neurosurgery. 2008;63:69-77.

18. Hoh DJ, Elder JB, Wang MY. Principles of growth modulation in the treatment of scoliotic deformities. Neurosurgery. 2008;63:211-21.

19. Kane WJ. Scoliosis prevalence: a call for a statement of terms. Clin Orthop Relat Res. 1977;126:43-6.

20. Kim HJ, Blanco JS, Widmann RF. Update on the management of idiopathic scoliosis. Curr Op Pediatrics. 2009;21:55-64.

21. King JD, Lowery GL. Results of lumbar hemivertebral excision for congenital scoliosis. Spine. 1991;16:778-82.

22. Kouwenhoven JWM, Castelein RM. The pathogenesis of adolescent idiopathic scoliosis: review of the literature. Spine. 2008;33:2898-908.

23. Lenke LG. The Lenke classification system of operative adolescent idiopathic scoliosis. Neurosurg Clin N Am. 2007;18:199-206.

24. Lincoln TL. Infantile idiopathic scoliosis. Am J Orthop. 2007;36:586-90.

25. McMaster MJ, Singh $\mathrm{H}$. The surgical management of congenital kyphosis and kyphoscoliosis. Spine. 2001;26:2146-55

26. Pahys JM, Guille JT, D'Andrea LP, Samdani AF, Beck J, Betz $R$. Neurological injury in the surgical treatment of idiopathic scoliosis: guidelines for assessment and management. J Am Acad Orthop Surg. 2009;17:426-34.

27. Pérennou D, Marcelli C, Hérisson C, Simon L. Adult lumbar scoliosis: epidemiologic aspects in a low-back pain population. Spine. 1994;19:123-8. 
28. Samdani AF, Storm PB. Other causes of pediatric deformity. Neurosurg Clin N Am. 2007;18:317-23.

29. Schwab F, Lafage V, Farcy JP, Bridwell K, Glassman S, Ondra $S$, et al. Surgical rates and operative outcome analysis in thoracolumbar and lumbar major adult scoliosis application of new adult deformity classification. Spine. 2007;32:2723-30.

30. Shaffrey $\mathrm{Cl}$, Wiggins GC, Abel MF. Pediatric spinal deformities. In: Benzel EC, editor. Spine surgery: techniques, complication avoidance, and management. Philadelphia: Elsevier Churchill Livingstone; 2005, p. 824-73.

31. Slosar PJ. Indications and outcomes of reconstructive surgery in chronic pain of spinal origin. Spine. 2002;27: 2555-63.

32. Smith JS, Abel MF, Shaffrey $\mathrm{Cl}$, Ames CP. Decision making in pediatric spinal deformity. Neurosurgery. 2008;63:54-68.

33. Smith JS, Fu KM, Urban P, Shaffrey Cl. Neurological symptoms and deficits in adults with scoliosis who present to a surgical clinic: incidence and association with the choice of operative versus nonoperative management. J Neurosurg Spine. 2008;9:326-31.

34. Smith JS, Shaffrey $\mathrm{Cl}$, Berven S. Improvement of back pain with operative and nonoperative treatment in adults with scoliosis. Neurosurgery. 2009;65:86-94.

35. Smith JS, Shaffrey $\mathrm{Cl}$, Berven S. Operative versus nonoperative treatment of leg pain in adults with scoliosis: a retrospective review of a prospective multicenter database with two-year follow-up. Spine. 2009;34:1693-8.

36. Smith JS, Shaffrey CI, Kuntz IV C, Mummaneni P. Classification systems for adolescent and adult scoliosis. Neurosurgery. 2008;63:16-24.
37. Soucacos PN, Zacharis K, Gelalis J. Assessment of curve progression in idiopathic scoliosis. Eur Spine J. 1998;7:270-7.

38. Takahashi S, Delécrin J, Passuti N. Surgical treatment of idiopathic scoliosis in adults: an age-related analysis of outcome. Spine. 2002;27:1742-8.

39. Thompson GH, Akbarnia BA, Campbell RM Jr. Growing rod techniques in early-onset scoliosis. J Pediatr Orthop. 2007;27:354-61.

40. Weinstein SL. Adolescent idiopathic scoliosis: prevalence and natural history. In: Weinstein SL, editor. The pediatric spine: principles and practice. Philadelphia: LippincottRaven; 1994, p. 421-9.

41. Weinstein SL, Ponseti IV. Curve progression in idiopathic scoliosis. J Bone Joint Surg (Am). 1983;65:447-55.

42. Winter RB. Congenital scoliosis. Orthop Clin North Am. 1988;19:395-408

43. Winter RB, Lonstein JE, Denis F. Pain patterns in adult scoliosis. Orthop Clin North Am. 1988;19:339-45.

\section{Endereço para correspondência}

Elton Gomes da Silva

Departamento de Neurologia - FCM/Unicamp

Rua Tessália Vieira de Camargo, 126

13083-970 - Campinas, SP, Brasil

E-mail: elgosi@yahoo.com.br 